\title{
Le migrazioni musicali della cantastorie Etta Scollo: trenta anni di canzoni nomadi tra i generi e le culture
}

\author{
Dagmar REICHARDT (Riga / Hamburg)
}

\section{Summary}

For decades the Italian singer and songwriter Etta Scollo, born in Catania in 1958, has been living in Germany after moving from Sicily to Turin, then to Vienna and touring for an intense period through the US and Europe - always coming back, though, to her home island in terms of creating music as well as of performing in public on site. In her musical work, the absence of a place is paradoxically intermingled with a strong attachment to it, as her compositions oscillate between classical music, jazz, and avant-garde pop. While telling personal stories and composing a transmedia-related mix of stylistic devices and poetic elements, Scollo's work displays an extraordinary openness with regard to other genres and cultures.

By recalling the migratory past of the Sicilian island and Catania -birthplace of the romantic composer and father of the bel canto Vincenzo Bellini (1801-1835), of Sicilian folk songs (including the cunti, the tarantella, etc.) and the traditional use of special instruments (such as scacciapensieri, ciarameddu, tambureddu, guatrara, etc.) - Scollo not only intends to highlight the suffering of Sicilian people, but also points out their unfailing poeticity and authentic aesthetic potential. Focusing on a total hybridity and transmitting a genuine and dynamic transcultural movement, her suggestive songs can be analyzed productively by referring to the nomadism first envisioned by the French poststructuralists Gilles Deleuze and Félix Guattari (Teatrise on Nomadology, 1986 [1980]). Scollo's texts describe and sing about an intriguing poetry of displacement and mobility, rooted in history and future-oriented at the same time. From this narrative and its empathic and syncretistic ethos emerges a musical oeuvre that is highly intellectual and spans thirty years of prolific performance to this day. Its stylistic integrity is based on various crossover devices and pastiche techniques which profess a different, postmodern freedom and deal with dislocation, musical migrations and with shifts into a global world, without homogenizing or reducing cultural values. 


\section{Musica 'tra' i generi e 'tra' le culture}

La cantautrice d'origine italiana Etta Scollo, nata il 27 maggio 1958 a Catania, vive ormai da più di due decenni in Germania, dopo essersi trasferita dalla Sicilia prima a Torino, per studiare l'arte e l'architettura, poi a Vienna, dove si è cimentata con il canto e il ballo al conservatorio, e dopo aver trascorso un periodo intenso di viaggi attraverso l'America e l'Europa, tornando però sempre - anche in termini musicali e di tournée - a casa in Sicilia. Nella sua opera musicale paradossalmente si uniscono l'assenza da un luogo e allo stesso tempo un fortissimo attaccamento ad esso. Le composizioni tra tradizione siciliana e pop d'avanguardia danno prova di una grande apertura per altri generi e altre culture, raccontando piccole storie personali e componendo un amalgama intermediale di stilemi e procedimenti poetici 'nomadi'. ${ }^{1}$

Ricordando infatti il passato migratorio dell' isola siciliana, patria del grande compositore romantico ottocentesco e maestro del bel canto Vincenzo Bellini (1801-1835), i suoi canti popolari (includendovi le filastrocche, i cunti dei cantastorie, la danza tradizionale della tarantella ecc.) e l'uso consapevole di speciali strumenti (scacciapensieri, ciarameddu, tambureddu o tammureddu, guatrara, chitarra classica, sassofono, cello, trombone, tuba, mandolino, fisarmonica, kinsho-koto, harmonium indiano, sega musicale, liuto rinascimentale, e via dicendo), Scollo non solo intende evidenziare le sofferenze della Sicilia ma anche l'inesauribile lirismo e il suo grande potenziale estetico. Focalizzando una totale ibridità e trasmettendo all'ascoltatore un genuino movimento ininterrottamente dinamico in veste transculturale, ${ }^{2}$ le sue canzoni rappresentano un nomadismo ideale come è stato prefigurato in formula paradigmatica dai post-strutturalisti francesi Gilles Deleuze e Félix Guattari a cavallo tra gli anni Settanta e Ottanta (Deleuze/Guattari 2005), descrivendo e cantando in modo tanto suggestivo quanto intenso una moderna poetica dello spostamento e della mobilità. Nasce così un'opera musicale fondata su diversi pastiche che professano una libertà diversa e postmoderna e che tematizzano la dislocazione e lo scivolamento in un mondo globalizzato, senza omogeneizzare o ridurre alcun valore culturale.

Con l'intento di definire più da vicino quale spirito 'nomade' pervada la musica di Etta Scollo, in questo studio rivisitiamo dapprima la sua carriera e il suo percorso biografico attraverso la lente dell'esperienza migratoria, andando ad fontes per correlare queste due categorie costitutive - biografia personale e percorso migratorio - ad alcuni aspetti della tradizione poetica e musicale in Sicilia in un primo sottocapitolo analitico (cf. "Storia di una migrazione siciliana"). Andando subito in medias res proveremo ad applicare le nostre riflessioni preliminari direttamente ad alcune canzoni scelte di Etta Scollo, mettendo in primo piano i suoi album Casa (2003) e Canta Ro' (2005) - inciso in collaborazione con l'Orchestra Sinfonica Siciliana, diretta da Angelo Faja, in omaggio a Rosa Balistreri - che segnano una cesura iniziatica nella sua opera.

Già in queste prime opere si intuiscono infatti i discorsi che Etta Scollo segue attorno alla sua ricerca antropologica, etnologica, storica e culturale del cosiddetto continente Sicilia. Incominceremo quindi con la presentazione dei suoi testi legati al tema della migrazione e 
delle sue composizioni cruciali, sempre considerandoli nell'ottica di una presumibile transculturalità ovvero di un nomadismo che crediamo vi si manifesti. Osserveremo come affermano la loro importanza in questo contesto i progetti Les Siciliens (2007) e Il fiore splendente (2008), prima di continuare con una breve rilettura dell'album Lunaria (2014), basato sulla favola teatrale omonima di Vincenzo Consolo. Infine, focalizzando Scollo con cello (2015) - un lavoro che Etta Scollo ha realizzato in collaborazione con la cellista Susanne Paul scopriamo un album sinergico che vive esplicitamente della mistura di generi musicali, dei media e delle diversissime connotazioni culturali, raggiungendo ormai un altissimo livello intellettuale e artistico.

Dopo poi aver riconsiderato al principio del nostro terzo sottocapitolo ("Parametri di una musica in movimento") l'approccio metodologico, allacciandoci ad alcuni teoremi transculturali che completano un avvicinamento sistematico all'opera musicale in questione, verifichiamo le nostre ipotesi tramite una critica approfondita dei brani introdotti nel capitolo precedente. Come le analisi dimostrano, quel che compone il nucleo dell'opera di Scollo in sostanza sono soprattutto l'intensità straordinaria e la bravura poliedrica con le quali l'artista riesce a creare una musica dinamica, movimentata e spinta dall'impeto vitale di migrare, riempiendo gli spazi vuoti 'tra' le tonalità, 'tra' i suoni, 'tra' le melodie, le parole e la musica. Probabilmente l'effetto che ne risulta è così naturale non solo grazie al suo grande talento, alla sua magnifica voce, al lavorio, alla diligenza, alla disciplina e al piacere e alla passione con le quali si dedica al mondo della musica e dello spettacolo. Ma in più, Etta Scollo riesce a trasmettere al suo pubblico internazionale anche un suggestivo messaggio artistico costruito 'tra' i paesi (Germania, Austria, Italia, l'America della cultura jazz, i Paesi arabi, ecc.) che ha portato a una musica segnata dalle più diverse tradizioni e tecniche di rappresentazione, cioè quelle del pop, del jazz, del rap, della musica rinascimentale, musica classica e/o popolare, dell'uso di elementi letterari e di poesia, danza e ballo nella pratica musicale, dell'esibizione teatrale melodica, dell'inclusione compositiva delle arti figurative, del disegno, film e video, e via dicendo. L'artista ha così inventato un microcosmo strettamente attaccato al suo proprio e autentico profilo all'interno della sua funzione da cantante, da compositrice e da cantautrice ovvero da cantastorie.

Come vedremo, essere in movimento, spostarsi variando le morfologie artistiche e gli approcci critici caratterizza sia il procedimento da lei adoperato, sia il messaggio etico che ci propone. La sua opera, vista integralmente, pratica proprio quelle procedure relative a una localizzazione della musica in un contesto di globalizzazione che intendeva Homi K. Bhabha (cf. Bhabha 1994) quando parlò dell'essere in-between, accennando cioè a una nuova collocazione della cultura nel cuore delle nostre società che identificò con il cosiddetto 'terzo spazio', the third space, nel quale, sempre secondo Bhabha, si crea 'la' cultura tout court-e anche l'impegno civile di Scollo come vedremo nel nostro capitolo conclusivo. 


\section{Storia di una migrazione siciliana}

È proprio Etta Scollo come creatrice di una cultura ibrida così affine alla nostra esperienza quotidiana che affascina dal primo momento lo spettatore e l'ascoltatore della sua musica. Da un punto di vista transdisciplinare, postmoderno e nomade, ciò si manifesta nel senso che, tanto le sue melodie, quanto lei stessa nella sua dimensione più intima, siano coinvolte in un perpetuo movimento vitale e vitalizzante che ci spalanca le porte ad un mondo emozionalmente 'altro', diverso, sospeso in un affascinante spazio di penombra in-between, universalmente polifonico e musicalmente transculturale. Un carisma che deriva dalla sua toccante espressività artistica, tanto sul palcoscenico quanto tramite le incisioni discografiche, $\mathrm{e}$ la sua convincente integrità che genera un modello per il soggetto transculturale, innovativo e cosmopolita per eccellenza, andando oltre i generi e le etnie e incoraggiando uno sviluppo positivo ed energico che si rispecchia sia nella sua musica, sia nella sua stessa vita.

Nel 1983 la musicista di origine catanese, figlia di un avvocato anti-mafia di posizione progressista, e dal 2005 residente a Berlino, vince il primo premio al Diano Marina Jazz Festival, allora diretto dal noto jazzista Giorgio Gaslini (1929-2014). Solo cinque anni più tardi, nel 1988 esce la sua hit pop "Oh Darling"3 (Scollo 1988) che è una cover del brano di successo dei Beatles (periodo di attività musicale: 1960-1970), composto da John Lennon e Paul McCartney nel 1969 e adattata da Scollo in lingua italiana, con cui riscuote un successo che le vale non solo il disco d'oro ma anche varie settimane in testa alla hit parade austriaca. Negli anni successivi seguono diverse tournée di Scollo nei club in Austria, Germania e Svizzera, dove ben presto riesce a farsi un nome, inizialmente seguendo la musica pop di stampo austriaco, già allora da lei contaminata da elementi estranei, sovversivi, ambigui. Fino a oggi, la sua musica è un mix di musica tradizionale italiana fusa con il soul, con le pratiche della musica classica e con altri stilemi e procedimenti poetici come la "JazzFusion, il Funk, Punkrock, elementi mediterranei, arrangiamenti polifonici, strutture minimalistiche, visualizzazioni simboliche" (Reichardt 2007, 351) ${ }^{4}$, e sempre unite ad un parlato, costruito sulla recita di parole, sullo storytelling e sul racconto lirico-poetico.

In genere i brani di Scollo abbinano agli influssi autobiografici, che li rendono così credibili, convincenti e appassionati, una certa ambivalenza, che spesso si nutre del tentativo di trasferire il senso, i contenuti e l'anima delle sue canzoni in un'altra sfera. Creano un altro linguaggio, alternando parole, melodie, strumenti specifici e narrazioni sceniche a esclamazioni ludiche, al portamento corporeo, al ballo espressivo e alla mimica drammatica, a un cambio di costumi, di sceneggiatura o dei media, e a tante altre variazioni giocose, teatrali e contenutistiche. Confessando la fragilità dei suoi pezzi musicali e condividendola con il suo pubblico, Etta Scollo trasforma le debolezze in punti di forza. Il vigore semiotico che ne emerge mette in moto una sinergia che diventa un linguaggio musicale autonomo, una estetica indipendente, sciolta e aperta all'altro che caratterizza non solo la comunicazione tra individui civilizzati ma che rende unico anche il suo stile ibrido, la sua filosofia transculturale e il suo libero modo di essere, plasmando un vero e proprio 'pianeta Scollo' pieno di associazioni estetiche dell'alterità, di rotture ritmiche e ideali umanitari: 
Um etwas [...] zu entwickeln, was der eigenen Ästhetik entspricht, und auch die Ideale, die Gedanken, das Kulturelle, was man mit anderen Menschen teilt - also man befindet sich quasi dann in einem Land von verschiedenen Kulturen, die aber teilen eine gemeinsame Sprache. Und diese gemeinsame Sprache ist die Kunst, die Musik, ist die Art, mit der man umgeht mit anderen Menschen - diese Offenheit gegenüber anderen Kulturen. ${ }^{5}$

Negli anni Novanta, la cantautrice e cantastorie di origine siciliana si trasferisce a Amburgo dove la sua carriera musicale prende una nuova direzione: fonda con altri musicisti un gruppo musicale sotto il nome di Etta Scollo e si esibisce in un suo primo grande concerto allo Star-Club, che è il locale dove avevano suonato anche i Beatles durante il loro periodo tedesco, quando ancora pochi conoscevano questo giovane gruppo venuto da Liverpool, che con la Beatlemania scriverà storia musicale. Scollo si immerge in diverse sperimentazioni con la musica contemporanea, interessandosi anche alla musica da film contribuendo il brano "I tuoi fiori" al film Bad Guy (2001) del regista cinematografico sud-coreano Kim-Ki-Duk, nato nel 1960 a Bonghwa e quindi quasi coetaneo di Scollo. Dopo aver registrato con la London Session Orchestra diretta da Will Malone l'album Blu (1999) e venendo originalmente dal pop, in Germania Scollo si apre sempre più al jazz, al soul e a sequenze sonore insolite a partire dall'album Casa (Scollo 2003).

Casa è seguito dall'album Canta Ro'(Scollo 2005), il cui titolo indica l'imperativo "Canta Rosa!" che fu allo stesso tempo l'invito del poeta Ignazio Buttitta (1899-1997) rivolto alla cantante folk siciliana Rosa Balistreri (1927-1990) di cantare in dialetto siciliano, imperativo culturale ripreso poi appunto da Etta Scollo per il suo progetto sull'opera della compaesana sanguigna siciliana, che - forse non solo secondo l'anagrafe, ma anche spiritualmente avrebbe potuto essere sua madre. Infatti Scollo si dedica in questa raccolta di canzoni al patrimonio spirituale e musicale di Rosa Balistreri, vincendo con l'album diversi premi (Premio Pino Veneziano 2005, Weltmusikpreis Ruth 2007, Premio Rosa Balisteri Alberto Favara VIII 2008) e dirigendo l'attenzione del pubblico verso questa straordinaria protagonista della canzone popolare siciliana. Il progetto culturale Canta Ro' susciterà grazie a Scollo un mini-revival di Rosa Balistreri e del suo patrimonio musicale, portando a una registrazione storica non solo dei pezzi di Balistreri stessa (Balistreri 2007), ma anche a una seconda incisione dal vivo di Etta Scollo nel 2006 (Scollo 2006). La passione di Scollo per l'indagine della storia culturale, gli sfondi etnici, i discorsi femminili e i dialetti della Sicilia, per poi rianimarli nel palcoscenico musicale su un livello transculturale, si accentua nell'album Les Siciliens (Scollo 2007) che contiene canti dal vivo di storie, poesie e leggende siciliane in lingua siciliana.

Anche le ultime due canzoni di Casa - l'album che segna appunto una svolta estetica significativa all'interno dell'opera di Scollo nel 2003 - sono cantate in siciliano: "Suspira" (composizione di Rosa Balistreri) e "Unn’è" (Scollo 2003). Nell'album sono ricorrenti i temi dell'assenza, della malinconia e della ricerca delle proprie radici, che l'io lirico ritrova 
nell'ubiquità, concetto universale che si condensa nel lessema esemplare 'ovunque' come risulta dalla canzone "Casa" che dà il titolo all'album:
Là è la mia casa
la terra natìa
là mi ritrovo accovacciata
nel grembo della mia malinconia
$[\ldots]$
Un rifugio per l'integrità,
un posto per l'orgoglio
un rifugio

Là è la mia casa...

Ovunque vado

ovunque canto

ovunque guardo

ovunque sento casa

Là è la mia casa...6

Nel quarto brano intitolato "Assente" la sensazione di smarrimento si rivolge a un 'tu' ovvero a una seconda persona singolare ambivalente, ignota o comunque non nominata. I parametri emozionali, affettivi, da un lato, e quelli locali, geografici o topografici, dall'altro lato, si intrecciano e si sovrappongono. L'io canta:

Sei nato già cresciuto

già comprato, già venduto,

non sei e non sai,

non puoi e non fai

ne [sic!] padre ne [sic!] futuro

soltanto il tuo respiro

è il ritmo del presente

nascosto fra la gente

assente, per sempre assente.

Ed io ti vedo e ovunque ti sento

sei dentro me

inesorabilmente e sempre 
L'ascoltatore non sa se l'apostrofe sia indirizzata a una persona amata (ovvero a un amante), oppure a un luogo personificato (ovvero alla Sicilia). Scollo accentua questo dubbio collegato alla sua propria identità ibrida in Canta $R o^{\prime}$ ' cantando per la prima volta tutti i testi in siciliano, volendo salvare il vasto e variopinto patrimonio culturale della sua isola, tramandando le tradizioni della lirica popolare siciliana. Dall'altra parte Scollo intende rivisitare, reinventare e reinterpretare l'opera della cuntista Rosa Balistreri in ottica postmoderna ovvero transculturale mantenendone l'impronta dialettale. Infatti il progetto della Scollo è intento a ricordare non solo le canzoni siciliane interpretate da Balistreri, ma anche l'impegno della cantastorie: i testi poetici conservati e attualizzati dalla Scollo servono da archivio poetico e orale per illustrare e far circolare il patrimonio culturale, lessicale e artistico della Sicilia, aperto in varie direzioni etniche, linguistiche, mediali, interdisciplinari e stilistiche. Le ballate riprendono fra l'altro le tradizioni dei contadini che sono profondamente ancorate nel popolo e nella cultura della Sicilia allargandole per esempio nel settimo titolo dell'album, "I pirati a Palermu" (Scollo 2005), composto da Ignazio Buttitta e Rosa Balistreri, su tutte le canzoni nate nelle prigioni. Interpretando "quel manifesto di Ignazio Buttitta contro dominazioni e speculazioni" che vuole essere, appunto, il testo de "I pirati a Palermu", Etta Scollo corregge così l'immagine stereotipata di una Sicilia fatalistica, sacrificata, sottomessa o malavitosa trasferendo con l'aiuto autorevole dell'Orchestra Sinfonica Siciliana il mondo espressivo della Balistreri senza cadere in una scontata imitazione. In tal modo Scollo riesce a produrre un palinsesto orientato alla corrente folk in Italia degli anni Sessanta e a realizzare una virtuale e reale collaborazione ovvero un lavoro di rianimazione e ristratificazione del passato inventando una pretenziosa reécriture postmoderna del testamento spirituale di Rosa Balistreri, come dimostra l'ultima canzone dell'album, "Quannu moru" ("Quando io muoio"):

Quannu jù moru cantati li me canti 'un li scurdati cantatili pi l'antri

Quannu jù moru pinsatimi ogni tantu ca pi sta terra 'n cruci murivu senza vuci

Quannu jù moru purtatimillu un ciuri un ciuri granni e russu cumu lu sangu sparsu ${ }^{8}$ 
Il lavoro di Etta Scollo risponde alla preghiera della Balistreri espressa nella prima strofa qui sopra riprodotta e che tradotta in italiano significa: "Quando io muoio / cantate i miei canti / non li scordate / cantateli per gli altri." La reinterpretazione della Scollo e la tradizione orale delle parole della Balistreri impediscono l'avverarsi dell'oscura sua profezia "che per questa terra in croce / sarò morta senza voce" ("ca pi sta terra 'n cruci / murivu senza vuci"). La Scollo smentisce questo cattivo esito dedicando alla Balistreri, simbolicamente parlando, "un ciuri" ("un fiore") postumo che le rende omaggio senza negare la storia del suo paese ovvero il passato doloroso della Sicilia.

Le sue interpretazioni dimostrano vivamente che, pensando alla domanda retorica formulata dalla femminista tra i teorici del postcolonialismo, Gayatri C. Spivak, nel titolo del suo saggio Can the Subaltern Speak? (1988), i subalterni possono 'parlare' eccome. L'intonazione essenziale, urgente e nostalgica della Scollo evidenzia le sofferenze della Balistreri e della Sicilia in generale, ma l'aspetto letterario della Sicilia vissuta come assenza paradossale o come desiderio interrotto, nella musica della Scollo non produce assolutamente più un movimento a ritroso (come invece lo troviamo nei testi dei classici Poeti della nostalgia ${ }^{9}$ ). $\mathrm{Al}$ contrario: con la sua accanita ricerca delle origini del paese di Trinacria, delle sue usanze, del suo mito storico, del suo paesaggio pittoresco e della sua estetica postmoderna, Scollo riesce a rappresentare una totale ibridità e a trasmettere un genuino movimento ininterrottamente dinamico in veste transculturale.

Un anno dopo aver inciso l'album Les Siciliens, nel 2008 Scollo, concentrandosi ancora sulle composizioni di musica tradizionale, crea il progetto poetico-musicale "Il fiore splendente" (Scollo 2008) che rende omaggio ai poeti arabi-siciliani del IX-XII secolo e al quale partecipano grandi esponenti del mondo della musica pop e contemporanea, quali Franco Battiato, Giovanni Sollima, Markus Stockhausen e Nabil Salameh. Negli anni 2009 e 2010 Scollo si misura invece con altre attualizzazioni transmediali di progetti classici: recita la parte di Elena nella rappresentazione musicale del Faust II di Johann Wolfgang von Goethe e il ruolo di Alice nell'opera Alice nel paese delle meraviglie al Teatro Massimo di Palermo. Inoltre rielabora la musica di Giuseppe Verdi per una moderna messa in scena di Rigoletto rappresentata per la prima volta alla Neuköllner Oper a Berlino nel 2009.

Dopo l'escursione letteraria della cantautrice con l'album Lunaria (Scollo 2014), che è un adattamento musicale del racconto teatrale omonimo Lunaria (1985; Premio Pirandello 1985) scritto dall'autore Vincenzo Consolo (1933-2012), Scollo si esibisce come duo in Scollo con cello. Tempo al tempo (Scollo 2015) insieme alla violoncellista sud-californiana con origini tedesche e messicane Susanne Paul, nata nel 1970 in California e oggi residente a Berlino, come Scollo. Chiaramente traspare da questo ultimo album, quanta strada abbia fatto l'artista catanese-tedesca dalla lontana cover di "Oh Darling" del 1988. In Scollo con cello combina in maniera sciolta il folklore italiano con quello arabo, la chanson con la musica rinascimentale, ritmi ballabili con pezzi filosofico-dadaisti (come l'ironico brano decostruttivista "Derrida"). ${ }^{10}$

Ciò non toglie niente all'attaccamento che Scollo prova nei riguardi della 'sua' Sicilia, isola che ha una lunga tradizione di poesia e musica. È sufficiente ricordare la Scuola poetica 
siciliana del Duecento, quando il poeta Giacomo da Lentini (ca. 1210-1260) fu attivo alla corte di Federico II, che coltivò un ambiente altamente culturale e plurietnico, preparando il terreno al Dolce Stil Nuovo, epoca che canonicamente segna gli esordi della letteratura italiana. Su questa base tanto antica quanto feconda nel Novecento transculturale moltissimi nomi di scrittori siciliani si fanno avanti - da Luigi Pirandello (1867-1936), insignito del Premio Nobel per la letteratura nel 1934, a Salvatore Quasimodo (1901-1968), Premio Nobel nel 1959, ai poeti Ignazio Buttitta e Lucio Piccolo (1901-1969), e agli scrittori Leonardo Sciascia (1921-1989) e Vincenzo Consolo, Premio Strega 1992, appunto. La musica siciliana ha tante sfaccettature come l'opera musicale di Scollo stessa: a partire dall'opera lirica del suo concittadino catanese Vincenzo Bellini per arrivare ai balli popolari meridionali (come la tarantella, la pizzica, ed altri), ai Canti popolari di carcere e mafia (1976) - raccolti e recuperati dal patrimonio culturale della Sicilia contadina e rurale ottocentesca da parte del poeta ed etnoantropologo Antonino Uccello (1922-1979) -, agli strumenti tradizionali e ai canti popolari che conservano ancora oggi parole e testi dialettali. Da qui si delinea il percorso transculturale che prende il lavoro artistico della migrante Etta Scollo, che rispecchiando tutta l'attualità di una Sicilia come metafora (Sciascia 1979) cerca di cogliere nei suoi singoli album e progetti di ricerca il Sud come emblema delle sue sofferenze e forse di un certo vittimismo, della povertà dell'isola, dei sincretismi e dell'emigrazione vissuta, ma anche simbolo del ritorno al paese natale, della sopravvivenza, resistenza vitale, storia multietnica e perenne fonte di creatività innovativa.

\section{Parametri di una musica in movimento}

Verifichiamo ora attentamente come gli album nominati e/o alcune singole canzoni nomadi di Scollo riflettano parametri transculturali che sono stati ulteriormente precisati o dall'idea contrappuntistica di Fernando Ortiz (Ortiz 1995), o dalla nomadologia di Deleuze e Guattari (nel loro Milles plateaux. Capitalisme et schizophrénie del 1980), o ancora dalla nozione dell'ibridità di un 'terzo spazio' di Homi K. Bhabha (Bhabha 1994), o anche dall'assioma dell' ubi bene, ibi patria di Wolfgang Welsch (Welsch 1999) ovvero quello di una 'modernità liquida' espresso da Zygmunt Bauman (Bauman 2000). Essendo questi teoremi tutti quanti approcci validi per riconoscere delle caratteristiche che potremmo definire transculturali, a noi qui deve interessare in primis quali morfologie transculturali elaborino le opere di Etta Scollo in dettaglio.

Il concetto di Welsch, il quale afferma che noi tutti siamo esseri ibridi e secondo il quale 'dove ci sentiamo bene, ci troviamo a casa' (ubi bene, ibi patria), lo riscontriamo subito nell'album Casa del 2003. Rianimando accuratamente il retroscena siciliano e il topos della terra madre (ovvero della lingua e/o musica 'madre') Casa si rifa alla tradizione siciliana e corrente letteraria della Poesia della nostalgia tramandataci originalmente dal noto poeta arabo-siciliano Ibn Hamdis (tra XI e XII secolo). È proprio sulla poesia araba che ancora anni dopo, nel 2011, porrà il suo accento il compositore contemporaneo Franco Battiato 
mettendo in musica alcune opere di Ibn Hamdis in occasione del suo progetto musicale Diwan: l'essenza del reale, e poi collaborando nel tour del 2013 con Etta Scollo, che con la sua voce lo accompagnerà assieme al cantautore di world music, artista e giornalista palestinese Nabil Salameh, che dal 2007 è cittadino italiano con decreto presidenziale, residente in Puglia. ${ }^{11}$ Ma già prima, nel 2003, trovandosi ormai da molti anni all'estero, lontana dalla sua 'casa' ovvero dalla sua terra, la cantante catanese in questo suo primo album che riflette seriamente su questioni identitarie sul piano culturale, dal titolo Casa, ${ }^{12}$ appunto, nella canzone intitolata "Ricordo" (cf. Scollo 2003) ${ }^{13}$ si riallaccia al topos della 'base sicura', della città natale ovvero alla metafora migratoria della 'casa'. Senza dover qui sottolineare la grande tradizione storica di questo tema classico, che iniziando dall' Odissea di Omero (ca. VIII-VII secolo a. C.) attraversa i secoli fino ai giorni nostri, in età postmoderna è invece utile qui ricordare brevemente l'esordio letterario della giovane scrittrice somalo-italiana Igiaba Scego. La sua storia personale è - pur essendo sostanzialmente diversa e né culturalmente e geograficamente né biograficamente congruente - comunque per certi versi fondamentalmente paragonabile a quella di Scollo, specie in riguardo al suo quarto romanzo, vincitore del Premio Mondello 2011, che porta il titolo significativo La mia casa è dove sono (Scego 2010), seguendo e specificando quindi sul piano informale il titolo Casa scelto dalla stessa Scollo.

La scrittrice italofona Scego - immigrante italiana di seconda generazione, nata a Roma nel 1974, (come Etta Scollo) appartenente a due mondi diversi che segnano la sua identità culturale, ovvero Roma e l'Africa - ricorda le molte storie che le aveva trasmesso sua madre nomade, rimasta in Somalia per ben due anni durante la guerra civile succeduta alla dittatura di Siade Barre in Somalia, lontana dalla figlia Igiaba. A livello teorico-letterario il significato centrale della metafora della casa in relazione alla migrazione è stato poi ampiamente studiato da critici come Jennifer Burns (Burns 2013), che dedica il terzo capitolo del suo libro proprio alla categoria della 'casa' ("Home"), o anche da Nora Moll (Moll 2015), che ne evince il nesso con la letteratura e la transculturalità nell'Italia contemporanea, rivelandone la funzione iconica che aveva ispirato anche Scollo a produrre il suo album chiave intitolato consapevolmente Casa già una decina di anni prima.

Dopo questo primo tentativo di delineare le proprie origini attraverso il linguaggio musicale, Etta Scollo continua - come già abbiamo accennato - la sua ricerca identitaria confrontandosi e identificandosi con la Cantatrice del Sud (Balistreri 1973) Rosa Balistreri (1927-1990), nata a Licata e scomparsa a Palermo nel 1990 dopo una carriera costruita fra enormi problemi esistenziali eppure accompagnata da grandi soddisfazioni professionali e giudizi molto positivi della critica. Come ricorda Scollo nel suo successivo album Canta Ro' (Scollo 2005), Rosa Balistreri, che proveniva da un ambiente siciliano proletariato in grandi difficoltà economiche, incontrò per la prima volta il poeta Ignazio Buttitta di Bagheria nel 1963 a Palermo durante la Settimana internazionale di nuova musica proprio nell'anno in cui si formò il famoso Gruppo 63. Rosa era affascinata da quell'ambiente culturale, e Buttitta, curioso della sua presenza, una sera esortava la donna dalla voce rauca e potente a cantare davanti agli amici intellettuali e artisti con l'incoraggiamento: "Canta Ro'?" Il poeta 
siciliano, all'epoca, rimase sbalordito dal "suo canto strozzato, drammatico, angosciato", come ricorda lo stesso Buttitta nel 1984 (cf. il booklet di Scollo 2005). Quarantadue anni più tardi, arrivati al Terzo Millennio, e ricordando la fondamentale tecnica mimetica del cover che con "Oh Darling" l'aveva portata al successo, Etta Scollo ricanta le canzoni dialettali di Balistreri in siciliano, prima accompagnata dall'Orchestra Sinfonica Siciliana con la quale ha curato il progetto (Scollo 2005), poi insieme al trio composto da Frank Wulff, Hinrich Dageför e dal fratello Sebastiano Scollo (Scollo 2006), trattandosi in ambedue i casi di registrazioni dal vivo, comunque non interamente riprese nell'auditorio. Certamente Scollo aveva coscienza della forza e dei contenuti espressi dalle canzoni della Balistreri come conferma la scelta di interpretare la canzone "I pirati a Palermu", ${ }^{14}$ cantata da Rosa Balistreri ed incisa sul suo album Rosa canta e cunta. Rari e inediti (Balistreri 2007), per poi essere ripresa e reinterpretata da Etta Scollo nell'album Canta Ro'.

Prima di aver partecipato al primo spettacolo di canti popolari $C i$ ragiono e canto diretto da Dario Fo nel 1966, Balistreri si era esibita al Festival della Canzone Popolare di Salerno nel 1964 contribuendo a dare forza al movimento del folk revival negli anni Sessanta alla riscoperta del genere musicale della canzone popolare non solo per sé, ma anche per il suo pubblico e per molti anni a venire. ${ }^{15}$ Dopo due pubblicazioni discografiche dedicate a Rosa Balistreri (Scollo 2005 e Scollo 2006) e al suo modo rivoluzionario di musicare, volendo anche storicamente onorare il "vero e proprio testamento spirituale" (cf. Scollo 2005, booklet) di Balistreri, Etta Scollo si riaggancia proprio al revival della musica folk siciliana di quel tempo per immergersi ancora nella sua ricerca sul popolo siciliano e sulla 'sua' Sicilia, sempre lontana, sempre presente, nei successivi due album: prima con Les Siciliens nel 2007, poi con Il fiore splendente nel 2008. Mentre il primo album è intitolato in lingua francese Les Siciliens, visto che combina il ritmo spensierato della chanson con qualche titolo popolare italiano interpretato nella tonalità densa, talvolta malinconica di diverse ballate $\mathrm{e}$ di canzoni in dialetto siciliano (cf. Scollo 2007) ${ }^{16}$, Il fiore splendente si apre alla cultura araba, dedicando all'antico poeta della nostalgia per eccellenza Ibn Hamdis una canzone intitolata programmaticamente "A Ibn Hamdis" (cf. Scollo 2008). ${ }^{17}$

Allargando l'approccio contrappuntistico tra la Sicilia e le sfere francofone e quelle arabe, e lavorando ancora in particolar modo sulle ibridità musicali e letterarie intrinseche nella letteratura postmoderna, Scollo produce nel 2014 Lunaria (Scollo 2014), che è un altro album-progetto siciliano-transculturale di origine teatrale-narrativa, dopo aver superato una pausa creativa che trova espressione nell'album intermediario Cuoresenza (Scollo 2011). ${ }^{18}$ Lunaria riprende il racconto omonimo pubblicato nel 1985 dallo scrittore siciliano Vincenzo Consolo - noto per i sui romanzi Il sorriso dell'ignoto marinaio (1976) e Nottetempo, casa per casa (1992) che vinse lo Strega nel 1992 - il quale da parte sua si era ispirato alla poesia Lo spavento notturno (1826) di Giacomo Leopardi (1798-1837) e al testo L'esequie della luna (1967) del poeta Lucio Piccolo. La "favola barocca" di un "atipico sovrano"19 è ambientata in una Sicilia fantastica che sull'album di Scollo si riflette nel racconto accompagnato musicalmente dalla recita dell'attore Giuliano Scarpinato, come maestosamente documenta tra l'altro il brano "Nella gioia luminosa dell'inganno" 20 
Le dinamiche transculturali e transmediali, che si manifestano sempre più palesemente nell'opera di Etta Scollo man mano che passano gli anni e che il volume della sua produzione artistica si arricchisce, si rispecchiano ormai in modo inequivocabile nel suo ultimo album, inciso in duetto con Susanne Paul, dal titolo Scollo con cello. Tempo al tempo del 2015. ${ }^{21}$ Mentre la canzone "Derrida" a forma di parodia decostruisce sia musicalmente sia intellettualmente un complesso contesto filosofico sciogliendolo in pura melodia leggera e armonica, il testo altamente lirico del pezzo "L'ala del tempo"22 è tratto dall'omonima raccolta poetica dell'autore contemporaneo e politico siciliano Sebastiano Burgaretta del 1995, di cui ricordiamo anche diversi saggi sulla Sicilia usciti più tardi sotto il titolo Di Spagna e di Sicilia (2011).

L'interpretazione live travolgente di Scollo a Catania è stata registrata su videoclip mettendo in rilievo i fragili disegni in bianco e nero del giovane artista catanese Francesco Balsamo che interrompono visualmente le riprese cinematografiche dal vivo, avvicinando lo spettatore all'unione vibrante, animata e sinergica di musica, arte, letteratura, cinema e spettacolo. Nel videoclip uscito su YouTube parallelamente all'album discografico il cantautore e regista catanese Franco Battiato introduce la recitazione della canzone da parte di Etta Scollo, mettendo l'accento sulla congenialità tra la sua composizione e la poesia di Burgaretta, dichiarando il risultato una "meraviglia" che "l'unione dei due [media]" - la musica e la letteratura - rappresenterebbe. ${ }^{23}$ In tal modo questo clip combina in maniera sofisticata elementi da show musicale con lo stile documentario di una ripresa di un concerto dal vivo, ricorrendo sul piano transmediale contemporaneamente alla musica strumentale, alla voce cantata, all'atmosfera del luogo e del pubblico, al mondo della letteratura, recita lirica ed esibizione teatrale sul palcoscenico, alla ripresa filmica e all'arte figurativa, creando un misto di tecniche narrative diverse tra di loro ma che nell'insieme riproducono un'ottima qualità caleidoscopica, che proprio a causa dell'effetto polifonico e contrappuntistico nella loro coerenza compongono un lavoro artistico particolarmente espressivo, delicato e impegnativo. Pregio che il cantautore Battiato aveva riconosciuto già nel 2009 quando in occasione della $22^{a}$ Fiera Internazionale del Libro a Torino elogiò la "Splendente Etta" non solo come una "splendente siciliana", ma soprattutto come "la nuova stella del 'Crossover" (Battiato 2009).

\section{L'etica di un nomadismo transculturale: 'mare aperto' vs. 'mare chiuso'}

In conclusione potremmo dire che Etta Scollo si presenta intenzionalmente nella veste di una cuntista transculturale del Terzo Millennio, che mette in scena le proprie canzoni con energie autenticamente correlate alla Sicilia e al suo passato storico da una parte, mentre dall'altra parte l'artista si rivolge ai movimenti nomadi postmoderni tra le culture, tra i media, tra i luoghi, tra gli stili, tra i tempi e le arti, creando un contro-discorso musicale cosmopolita che contrasta il parametro siciliano di provincia rendendolo 'glocale'. Infatti la cantante non solo realmente vive 'tra' Berlino e la Sicilia, ${ }^{24}$ creando e materializzando nella sua concreta biografia un 'terzo spazio' ibrido in-between proprio come lo espone concettual- 
mente Homi K. Bhabha. Ma il suo tragitto musicale rispecchia anche un perenne percorso migratorio che fa tramutare il suo personaggio, la sua opera musicale e la sua presenza sul mercato creativo in un fenomeno culturale omnicomprensivo, migratorio, cioè tipicamente nomade. Secondo le direttive etiche di Deleuze e Guattari la nomadologia infatti da un lato cerca di proteggere e promuovere l'autonomia di culture presumibilmente minoritarie (come per esempio quella siciliana), e dall'altro lato intende formare un contro-discorso che si oppone al capitalismo ovvero al discorso egemonico esprimendosi invece a favore di uno svincolato pensiero dinamico, adattabile e versatile, che pienamente corrisponde allo stile musicale di Scollo che combina diversi generi musicali in un'unica canzone come, tra tanti altri, nel brano "I pirati a Palermu" (Scollo 2005).

Convertendo la teoria contrappuntistica di Fernando Ortiz (1881-1969) in una formula di composizione melodiosa e applicandola materialmente durante il suo musicare, Scollo usa la funzione centrale del contrappunto nel contesto della musica polifonica creando un concetto stilistico sfaccettato, collegato a un messaggio ponderato che professa l'equivalenza e l'indipendenza delle singole voci. Con questo appello comunicativo la 'cantatrice' trasmette all'ascoltatore un'idea di pluralità, polivalenza e multiformità che ben si inserisce in un quadro transculturale di una Europa che si augura federalista, unita nella sua diversità, e, allo stesso tempo, elastica e resiliente riguardo alle sue frontiere 'liquide' come direbbe Zygmunt Bauman - quindi sempre pronte all'incontro tra i popoli, tra le lingue e le culture, ovvero sensibili all'interscambio culturale e all'osmosi di correnti e controcorrenti, discorsi e contro-discorsi, a tanti modi di espressione e all'impiego di tutta una serie di stilemi vari.

Vediamo infine le diverse caratteristiche del nomadismo transculturale che comportano sia il personaggio Etta Scollo di per sé, sia la sua musica, sia il suo modo di vivere e di lavorare sul piano stilistico-creativo. Come risulta dalle nostre analisi è stato non solo possibile ma anche molto produttivo ricavare le radici identitarie dell'opera di Scollo dalle sfere situate 'tra' le sue relazioni locali e condizioni globali, ritrovandole infine in una identità 'glocale' più che suggestiva e stimolante, soprattutto paradigmatica, ossia esemplare per moltissime creazioni culturali e stili di vita postmoderni. Innanzitutto abbiamo visto che questo suo nomadismo transculturale si definisce attraverso il parametro della migrazione: Etta Scollo sia in veste di persona privata, sia nel suo ruolo di cantante conosciuta a un vasto pubblico - attraverso la sua musica si presenta come una cittadina del mondo ovvero una cittadina d'Europa, che si muove serenamente attraverso lo spazio Schengen, e la cui biografia, anzi, si è composta proprio grazie alla circolazione nei vari ambienti culturali europei (Italia Austria - Germania, e oltre). Infatti lavora in più di tre sfere culturali europee che confluiscono nella sua opera, ovvero nell'ambito della cultura italiana, austriaca, tedesca, siciliana, francese, inglese, includendovi la sfera araba e quindi promuovendo anche indirettamente il 'polilogo' (cf. Wimmer 2000) transreligioso, se pensiamo ai suoi album Les Siciliens prima e Il fiore splendente poi.

Secondo - oltre all'approccio migratorio - si rivela transculturale anche il sincretismo dell'opera poliedrica di Etta Scollo, non solo per via delle diverse aree linguistiche nelle (e con le) quali lavora, ma anche per le innumerevoli combinazioni dei generi e strumenti 
musicali che sceglie per i diversi album, brani e canzoni. La sua 'musica mosaico' testimonia chiaramente non solo il gusto postmoderno in chiave melodica, ma documenta anche la svolta transculturale del suo pensiero, che unisce il sincretismo siciliano - e quindi una sfera d'intersezione principalmente geografica e locale - con quello globale, appunto dando vita a uno stile genuinamente 'glocale' che è orientato simultaneamente alle categorie dello spazio e del tempo, cioè sia al parametro topografico che a quello dell'ubiquità.

Terzo appare transculturale, evolvendo l'idea del network proposta da Wolfgang Welsch (cf. Welsch 1999), l'intenso modo di realizzare una costellazione specialmente adatta ad ogni singolo progetto, ad ogni album e ad ogni concerto a livello di teamwork collaborativo. Questo modo di procedere è certamente tipico di molti gruppi musicali, ma nel caso di 'Etta Scollo' il suo nome in realtà cela decine e decine di collaboratori dietro le quinte, che in gran parte compongono l'équipe che sostiene i progetti di Etta Scollo sin dall'inizio della band, dimostrandone una certa sostenibilità ossia una costanza e solidità che persistono da ormai ben venti anni. In maniera personale e paritaria la 'cantatrice' in veste postmoderna presenta comunque anche altri membri del gruppo sul palcoscenico, collaborando ed interagendo intensivamente per le singole produzioni e a diversi livelli con vari colleghi che normalmente operano in altre costellazioni, provenienti dal settore artistico, logistico, economico e politico fin dal suo esordio. Anche in quanto al gruppo-target, il gruppo Etta Scollo si appoggia espressamente su una community sorprendentemente sensibile, attiva e attenta - particolarmente ma non solo in Germania - specie se pensiamo al ruolo comunicativo-democratico che Etta Scollo concede al suo uditorio con il quale cura un rapporto dialogico in pubblico $\mathrm{e}$, andando oltre, spesso stringe amicizia. Ma ciò vale anche in quanto riguarda la sua apertura verso collaborazioni esterne reali o virtuali con personaggi culturali noti, e qui basti ripetere alcuni nomi che abbiamo incontrato nel corso di questo saggio, come Franco Battiato, Kim-Ki-Duk, Vincenzo Consolo, Susanne Paul, l'Orchestra Sinfonica Siciliana o anche Leoluca Orlando (mediatore italo-tedesco culturale e politico siciliano, menzionato da Scollo tra l'altro nel suo booklet di Canta Ro'; cf. Scollo 2005), Markus Stockhausen, Nabil Salameh e tantissimi altri.

Contemporaneamente a questo spirito collaborativo, si sviluppa quasi automaticamente uno stile orientato all'intermedialità - cioè combinando diversi livelli d'espressione come quello definito attraverso il testo e la coreografia scenica con quello della musica strumentale, del canto, dell'antropologia culturale e via dicendo - e alla trans-temporalità ovvero diacronia, che nasce collegando pezzi, che stilisticamente rinviano al Rinascimento, con altri del Novecento, o altri ancora che sono frutto di un pensiero postmoderno o anche correlato alla globalizzazione ecc. Quest' ultima caratteristica è legata a una certa simultaneità di fattori stilistici che vengono rafforzati dall'intensità e tensione morale trasmessa al pubblico dal personaggio artistico Etta Scollo. Questa integrità del messaggio transculturale che è inerente alla musica di Scollo si nutre di una consapevolezza circa l'ibridità del dolore, causato da un trauma (come quello suscitato per esempio dal distacco dalla patria ovvero dalla migrazione), o circa la rappresentazione della Sicilia come modello di un sincretismo storico e della coabitazione pacifica di diverse etnie. 
Infatti Etta Scollo fa dell'ibridità collegata alla storia e alla cultura della Sicilia un pregio, modellandola nella sua fantasia musicale come un 'terzo spazio', immaginato in termini teoretici da Homi K. Bhabha a metà degli anni Novanta, giusto quando Scollo si avvia a formare una band che porterà il suo nome durante il suo periodo amburghese. La Sicilia come metafora (1979) sciasciana si materializza nell'opera di Scollo attraverso un paradigma nomade (secondo Deleuze e Guattari) disegnando dei confini 'liquidi' nel segno di una Liquid Modernity (2000) di Zygmunt Bauman, che sono intrecciati e coinvolti nel fenomeno della migrazione del Terzo Millennio. Che questo aspetto sia vitale per i principi artistici di Etta Scollo lo conferma in ultimo il suo progetto più recente dalla forte connotazione politica e che si connette all'installazione artistica di Heidrun Hannusch intitolata Lampedusa $361 .^{25}$

L'opera della curatrice e ideatrice tedesca Hannusch coordina sotto il titolo Lampedusa 361 un'esposizione fotografica contemporanea inaugurata a Dresda il 13 febbraio 2017, poi resa accessibile al pubblico nel corso dello stesso anno anche a Düsseldorf. L'idea centrale della mostra non a caso sorge nella parte orientale e quindi periferica della Germania come contro-discorso ai movimenti euroscettici dei populisti di destra. Esponendo le fotografie di Carsten Sander che riproducono le immagini delle tombe dei profughi annegati nel Mar Mediterraneo a causa della cosiddetta 'crisi europea dei rifugiati', i visitatori possono ricordare la dimensione dell'attuale tragedia. Ispirandosi all'iniziativa, Etta Scollo compone per l'inaugurazione di Lampedusa 361 a Dresda la canzone bilingue "Sconosciuto - unbekannt" (Scollo 2017), ${ }^{26}$ dedicata a tutti i profughi senza nome e dimora - ovvero 'sconosciuti' - che negli ultimi anni sono morti nel Mediterraneo e le cui ceneri riposano a Lampedusa, disperse sui vari cimiteri dell'isola. A questa canzone, presentata per la prima volta al pubblico il 12 febbraio 2017 alla Semperoper di Dresda, seguì un secondo pezzo musicale che la cantante catanese ha rappresentato per la prima volta a Düsseldorf l's luglio 2017, recitando un discorso testuale lungo dieci minuti che ripete le parole della sindaca di Lampedusa Giusi Nicolini. Nel novembre 2012 infatti, Nicolini si era rivolta all'opinione pubblica mondiale accusando furiosamente il fatto che sul cimitero dell'isola non c'erano più tombe sufficienti per sotterrare tutti i profughi annegati al largo di Lampedusa.

È proprio nel contesto della canzone contrappuntistica a più voci dal titolo italo-tedesco "Sconosciuto - unbekannt" e del suo impegno politico-umanitario per il progetto d'arte Lampedusa 361 che la cosiddetta 'voce della Sicilia' - "Stimme Siziliens" esprime esattamente l'empatia che costituisce una delle condizioni sine qua non della transcultura, condividendo sensitivamente il suo dolore e dando una voce alla sua emozione attraverso il canto. In verità, però, la cantante siciliana-berlinese, costruendo un ponte musicale tra l'Italia e la Germania che sono tra i Paesi più afflitti dalla crisi, segue incessantemente il suo cammino. Già fin dal principio della sua impressionante carriera Scollo ha dimostrato di avere una grande sensibilità autoriflessiva sul piano transculturale, come confessa ricordando i suoi anni verdi in Austria: "Io ero un controsenso vivente... una siciliana a Vienna non si era mai vista. Spesso mi sentivo fuori luogo." (Bassan 2012) L'autoironia si fa seria quando Scollo, che certamente da sempre ha saputo farsi compenetrare da altre mentalità, riesce a formulare delicatamente le relazioni interculturali con reciproche fecondazioni sociali tra la 
sfera italiana e quella tedesca con le parole espresse durante una intervista in Germania nel 2012:

Il pubblico tedesco ha seguito l'Italia sempre e ne ha seguito tutti i cambiamenti, anche politici, culturali, artistici. Ha sempre guardato all'Italia con interesse e curiosità, con un occhio diverso da quello dei francesi o degli inglesi, un occhio più vigile, più costante e anche più fedele.

[...] Oggi l'italiano ha perso quel senso esotico che aveva un tempo. Non è più l'immigrato in cerca di lavoro, non è più l'operaio della Volkswagen. In compenso, ha mantenuto quella spontaneità che ai tedeschi piace tantissimo: noi siamo quelli che aprono la porta a tutti, quelli che ti invitano a cena senza tanti complimenti. Loro vengono molto colpiti dalla nostra accoglienza, si divertono e, dopo lo stupore iniziale, si lasciano trascinare. Questa ventata di italianità dà a Berlino, la città in cui adesso vivo, una connotazione diversa, più internazionale. (Bassan 2012)

Questa spontanea presa di posizione illustra bene come Etta Scollo impersoni e trasmetta al pubblico internazionale proprio quel nomadismo transculturale che celebra anche cantando. Allo stesso tempo - ancora una volta in maniera contrappuntistica - Scollo dispiega un contro-discorso che si allontana dal capitalismo, che Deleuze e Guattari avevano definito patologia schizofrenica e che nel neoliberalismo degli anni Duemila pare aveva preso dimensioni ancora più estreme, per tornare all'inizio del Terzo Millennio alle radici e origini culturali del suo 'continente' Sicilia rendendole compatibili con i processi della globalizzazione. Se Andrea Camilleri ha differenziato nel suo libro-intervista La testa ci fa dire (Sorgi 2000) tra i siciliani "di scoglio" e quelli "di mare aperto" - definendo quest' ultima categoria come coloro che decidono di utilizzare la loro provenienza siciliana per "vivere una vita diversa", per "proiettarsi su un altro orizzonte", affascinati "dagli estremi, dall'avventura, dal rischio, dalla ricerca dell'ignoto" con l'intento di lavorare su di sé e di imparare a mettersi "interiormente in movimento", spinti dalla voglia di scoprire come si fa "a diventare un errante, un nomade, a riprendere movimento, a sentire di non avere più dimora” e scoprendo infine che, come ammette Camilleri, "il mondo intero è ormai la mia casa" 28 - in tal caso possiamo sicuramente dedurre che Etta Scollo, come il suo compaesano Camilleri, non appartenga di certo alla categoria di un 'mare chiuso' o 'di scoglio', ma che sia da considerare francamente una siciliana 'di mare aperto'.

Infatti nel periodo tra il lontano 1988, quando trenta anni fa Etta Scollo debuttò in Austria con "Oh Darling”, e l'anno 2018 nel quale l'ingegnosa cantastorie compie i sessanta anni il 27 maggio, ${ }^{29}$ questa innovativa e prodigiosa musicista piena di talento, di saggia estroversione ed energetico virtuosismo ha sviluppato non solo una sua personale storia di migrazione, ma anche una compatta, diversificata e complessa narrazione musicale per esprimere una propria interpretazione dell'impostazione filosofica inizialmente proposta dai post-strutturalisti Deleuze e Guattari. È così che con una trasparente facilità e una profonda 
leggerezza, continua a visualizzare e a trasmetterci in diretta le linee di una estetica polifonica prodotta da un nomadismo transculturale niente 'scoglio' e tutto 'Scollo'.

\section{Note}

1 Con l'aggettivo 'nomade' e derivati mi riferisco principalmente al saggio sul nomadismo di Gilles Deleuze e Félix Guattari apparso in francese nel 1980 come dodicesimo capitolo di Mille plateaux. Capitalisme et schizophrénie (1980), che venne tradotto in inglese a parte, come libro separato, sotto il titolo Nomadology: The War Machine (1986) introducendo filosoficamente con più insistenza il concetto di nomadismo. Rosi Braidotti ha ripreso questo termine applicandolo esplicitamente alla condizione femminile nel 1992 (Braidotti 1992). Cf. anche i miei studi sulla scrittrice statunitense-indiana-italofona 'nomade' Jhumpa Lahiri (Reichardt 2017).

2 Per meglio definire il concetto del transculturale, della transcultura ovvero della transculturalità rimando ai seguenti titoli: in ambito italiano lo studioso romano di origine pugliese Armando Gnisci si è occupato sia del pensiero ibrido-postcoloniale (Gnisci 2003; Gnisci 2007), sia di quello transculturale (Gnisci 2012; Gnisci 2013), dopo che Le Pichon e Caronia avevano introdotto nel discorso accademico in Italia il termine della 'transcultura' già all'inizio degli anni Novanta (Le Pichon/Caronia 1991) e prima che Merlini e Fabiani l'abbiano ripreso in ambito letterario (Merlini/Fabiani 2016). - Il concetto è generalmente più noto in Germania grazie alle pubblicazioni di Wolfgang Welsch (Welsch 1999; Welsch 2017) che ne aveva anche subito sottolineato il nesso con i media assieme a Gianni Vattimo nel 1998 (Vattimo/Welsch 1998). Per l'uso del termine in relazione all'Italia e in particolare alla Sicilia si vedano inoltre i recenti studi critici di Kleinhans e Schwaderer (Kleinhans/Schwaderer 2013) e di Reichardt (Reichardt 2006; Reichardt 2018) - e in relazione al contesto europeo ovvero alla francofonia: Meinhof/ Triandafyllidou 2006; Reichardt 2017.

3 La hit "Oh Darling" è anche disponibile in una versione videoclip arrangiata all'epoca, nella cui estetica e narrativa filmica appaiono subito alcuni temi centrali della musica di Etta Scollo come gli arrivi, le partenze, gli addii, la nostalgia di casa, l'amore che si esprime in momenti di gioia, bellezza, libertà, erotismo, malinconia, vicinanza e lontananza, il desiderio di comunicazione e il fenomeno della migrazione in generale (si veda su YouTube: https:/www.youtube.com/ watch?v=MTRSShr2XOk).

4 Si vedano in Reichardt 2007 particolarmente le pagine 350-355 per ulteriori dettagli analitici riguardo l'opera di Etta Scollo.

5 Etta Scollo si esprime così in una intervista anonima, non firmata, uscita su YouTube il 24.5.2011 sotto il titolo poco specifico di Cuoresenza, ossia in occasione dell'uscita dell'omonimo album (si veda online su YouTube: https://www.youtube.com/watch?v=uZbNa1j5-pk; 1:52'-2:30'; consultazione 15.02.2018). Tradotto in italiano, questo commento associativo, espresso in un tedesco colloquiale, significa più o meno: "Per [... sviluppare qualcosa, che corrisponda alla propria estetica, e anche gli ideali, i pensieri, la cultura, che condividiamo con altre persone, tutto ciò ci porta in un Paese nel quale ci sono tante culture diverse, le quali tra di loro però condividono un 
linguaggio. E questo comune linguaggio è l'arte, è la musica, il modo come trattiamo gli altri - è proprio quest'apertura per le altre culture" (la traduzione dal tedesco in italiano è mia; D.R.).

6 I testi di tutte le canzoni - come anche quello del brano "Casa" - sono riportati nel booklet del CD Casa (Scollo 2003).

7 Cito dal booklet non paginato che accompagna il CD-ROM e che riporta commenti introduttivi di Pippo Ardini, Etta Scollo e Ignazio Buttitta, nonché tutti i testi delle canzoni in versione siciliana, italiana e tedesca (Scollo 2005).

8 Queste le ultime tre strofe della poesia intitolata "Quannu moru".

9 Le espressioni dei 'Poeti della nostalgia' (cf. Freni 1992, 14) o della 'Poesia della nostalgia' (cf. Granara 2000) vengono usate sempre più spesso in senso sinonimo alla tradizionale denominazione de 'I poeti arabo-siciliani' (Papa 1973) ovvero dei 'Poeti arabi di Sicilia' (Corrao 1987). Lo scrittore Melo Freni rapporta in questo contesto che "[c]on l'esilio coincise la fioritura dei poeti della nostalgia, tra i quali Ibn Hamdìs fu subito definito 'il più sublime”" (Freni 1992, 14). Anche Etta Scollo si ispirerà a questo poeta siculo-arabo dell'età normanna e al suo Canzoniere nella sua musica, come vedremo qui in seguito.

$10 \mathrm{Si}$ può ascoltare la canzone anche online: https:/www.amazon.de/Derrida/dp/ B00SF7ESDU/ref=sr_1_12? s=books-intl-de\&ie=UTF 8 \&qid =1491262208\&sr = 8 $12 \&$ keywords=scollo+con+cello (consultazione 15.02.2018).

11 Come segnala la stampa, la tour del progetto Diwan - l'essenza del reale di Franco Battiato è stata realizzata insieme a Etta Scollo e Nabil Salameh (voci) in novembre del 2013 passando per le città di Montecatini (PT), Parma, Genova, Ancona, Milano e Torino. Cf. http://www.radioitalia.it/news/franco_battiato/tour/6926_\%E2\%80\%9Cdiwan_\%E2\%80\%93_1_essenza_del_ reale\%E2\%80\%9D_franco_battiato_da_novembre_a_teatro.php (consultazione 15.02.2018).

12 L'album Casa è tematicamente profilato nel senso che Etta Scollo vi tratta, in un formato lirico compatto e in maniera condensata e concentrata, alcuni temi culturali esigenti e essenziali come la coesistenza di amore, vita e morte, il tema dell'assenza e della presenza, gli antipodi di arrivo e partenza, focalizzandosi sulla natura, sulla malinconia, sulla resistenza o anche sulla Sicilia come terra natia ecc. (cf. Scollo 2003).

13 La canzone si trova anche online: https://www.youtube.com/watch?v=DkQ3ZI7zb8Q (consultazione 15.02.2018).

$14 \mathrm{Si}$ confrontino le due interpretazioni vocali della canzone "I pirati a Palermu", cantata da Rosa Balisteri (https://www.amazon.de/I-pirati-a-Palermu/dp/B001R5KLHE/ref=sr_1_93 $? s=\mathrm{dmusic} \& \mathrm{ie}=\mathrm{UTF} 8 \&$ qid $=1518641263 \& \mathrm{sr}=1-93 \& \mathrm{keyword}=$ rosa + balistreri\&refinemen ts=p_n_format_browse-bin\%3A180849031) e da Etta Scollo (https:/www.youtube.com/ watch?v=O120lgEru98; consultazione 15.02.2018). - Si veda anche la registrazione dal vivo del concerto-omaggio Rosa! in occasione del 90esimo anniversario dalla nascita di Rosa Balistreri, presentato da Etta Scollo a Catania il 20.7.2017 al Chiostro di ponente del Monastero dei Benedettini, nel trio con Cathrin Pfeifer (fisarmonica) e Fabio Tricomi (plettri, percussioni e marranzano): https://www.youtube.com/watch?v=j2FXgVl7kw0 (consultazione 15.02.2018).

15 Conferma Pippo Ardini nella sua introduzione al booklet inserito nel primo album intitolato Canta Ro': "Ricordarsi di Rosa Balistreri e dei poeti, letterati, teatranti, 'cuntisti' e cantastorie che 
rappresentano il meglio delle nostre tradizioni popolari [...], ma anche di quanti tra gli studiosi (Pitré, Salamone Marino, Cocchiara, Vigo, Uccello, Favara, Feliciotto, ed altri) hanno speso tutta la loro vita per la conservazione a futura memoria delle generazioni a venire, è un dovere civile, oltre che sociale e culturale." (Scollo 2005)

16 Alcuni brani sono da ascoltare in parte anche online: https://www.amazon.de/siciliens-EttaScollo/dp/B00YD50E9U/ref=sr_1_1?s=dmusic\&ie=UTF8\&qid=1491260651\&sr=1-1-mp3albums-bar-strip-0\&keywords=scollo+les+siciliens (consultazione 15.02.2018).

17 Si veda inoltre l'intervista con Etta Scollo sul progetto Il fiore splendente, sull'importanza del viaggio e sulla genesi transculturale del suo disco: https://www.youtube.com/watch?v=4KAynAf0lXs. La canzone "A Ibn Hamdis" si può ascoltare sul sito di Etta Scollo: http://ettascollo.de/ ilfioresplendente/?lang=de (consultazione 15.02.2018).

18 In quanto alla sua crisi affettiva durante questa fase creativa si veda l'intervista che Etta Scollo ha rilasciato a Valerio Bassan in aprile 2012 a Berlino (cf. Bassan 2012).

19 http://ettascollo.de/lunaria/ (consultazione 15.02.2018).

20 Il brano è pubblicato non solo sul CD, ma anche sul sito di Etta Scollo: http://ettascollo.de/newsite/wp-content/uploads/2013/12/10.NELLA-GIOIA-LUMINOSA-DELLINGANNO.mp3 (consultazione 15.02.2018). - L'artista catanese descrive i suoi incontri personali con Vincenzo Consolo a Milano e l'intento del suo progetto in un'intervista dell'Associazione Musicale Etnea a Etta Scollo presso l'Università di Catania nel dicembre 2015 (online: https:/www.youtube.com/ watch?v=YXRanQAKZTk).

21 Durante il periodo della redazione finale del presente saggio nel maggio 2018 è uscito un nuovo album dal titolo Il passo interiore (Jazzhouse Records Inakustik JHR 151, 2018, CD) che ho presentato in lingua tedesca in un articolo online redatto in occasione del 60esimo compleanno di Etta Scollo (cf. Reichardt 2018).

22 Cf. la canzone "L'ala del tempo" registrata dal vivo a Catania e arrangiata in formato video-clip, con la presentazione di Franco Battiato e le illustrazioni di Francesco Balsamo online: https:// www.youtube.com/watch?v=KQ_LLqjuPP0 (consultazione 15.02.2018). - Etta Scollo commenta il progetto musicale, spiegando il nesso tra l'immagine psicologica del 'bambino interiore' e la categoria filosofica del tempo, anche in una intervista sempre rilasciata all'Associazione Musicale Etnea presso l'Università di Catania: https://www.youtube.com/watch?v=_f5213ladjU (consultazione 15.02.2018).

23 Si veda l'intervento introduttivo di Franco Battiato nel video-clip su YouTube: https://www.youtube.com/watch?v=KQ_LLqjuPP0 [0:00'-0:20'] (consultazione 15.02.2018).

24 Cf. la biografia sul sito dell'artista che termina con le parole: "Etta vive tra Berlino e la Sicilia", http://ettascollo.de/biografia/ (consultazione 15.02.2018).

25 Il sito della mostra Lampedusa 361 esiste attualmente solo in lingua tedesca: http://lampedusa361.de/lampedusa-361/ (consultazione 15.02.2018).

26 Etta Scollo ha eseguito la canzone "Sconosciuto - unbekannt" insieme a Susanne Paul (cello, canto), Cathrin Pfeifer (fisarmonica, canto) e Hinrich Dageför (percussione) che è stata archiviata online sotto al link della mostra Lampedusa 361: http://lampedusa361.de/installation/ (consultazione 15.02.2018). 
27 Cf. http://lampedusa361.de/installation/ (consultazione 15.02.2018).

28 Cf. "Siciliani di scoglio e di mare aperto", in: L'ermeneuta. Considerazioni inattuali, (27.03.2008), http://www.turicampo.it/2008/03/27/siciliani-di-scoglio-e-di-mare-aperto/ (consultazione 15.02.2018).

29 Ci tengo a ringraziare Etta Scollo per la sua apertura mentale e curiosità intellettuale, che ho avuto il piacere di conoscere e condividere per la prima volta durante un incontro dietro le quinte del Bucerius Kunstforum ad Amburgo nel 2004, serata alla quale è seguito un dialogo personale ininterrotto attraverso gli anni, che spero possa sfondare in un 'polilogo polifonico' in occasione di un'ulteriore collaborazione al convegno A.I.P.I. presso l'Università per Stranieri di Siena nel 2018.

\section{Bibliografia}

Bassan, Valerio: "Etta Scollo, la voce siciliana che incanta la Germania”. In: Linkiesta (01.04.2012), http://www.linkiesta.it/it/article/2012/04/01/etta-scollo-la-voce-siciliana-che-incanta-la-germania/5584/ (consultazione 15.02.2018).

Battiato, Franco: "Splendente Etta". In: La Stampa (15.5.2009), http://ettascollo.de/new-site/wpcontent/uploads/2012/11/La-Stampa15-5-2009.pdf (consultazione 05.06.2018).

Bauman, Zygmunt: Liquid Modernity. Cambridge: Polity Press, 2000.

Bhabha, Homi K.: The Location of Culture. New York: Routledge, 1994.

Braidotti, Rosi: "The Exile, the Nomad, and the Migrant. Reflections on International Feminism". In: Women's Studies International Forum 15,1 (1992), 7-10.

Burns, Jennifer: Migrant Imaginaries: Figures in Italian Migration Literature. Bern et al.: Lang, 2013. Corrao, Francesca Maria (ed.): Poeti arabi di Sicilia. Milano: Mondadori, 1987.

Deleuze, Gilles / Guattari, Félix: “Teatrise on Nomadology: The War Machine [1980/1986]". In: Deleuze, Gilles / Guattari, Félix: A Thousand Plateaus: Capitalism and Schizophrenia. Trad. e premessa Brian Massumi. London/Minneapolis: University of Minnesota Press, 2005, 351-423.

Freni, Melo: Il giardino di Hamdis. Palermo: Sellerio, 1992.

Gnisci, Armando: Creolizzare l'Europa. Letteratura e migrazione. Roma: Meltemi, 2003.

Gnisci, Armando: Decolonizzare l'Italia. Via della decolonizzazione europea n. 5. Roma: Bulzoni, 2007.

Gnisci, Armando: "Manifesto transculturale (aggiornato)". In: Rivista internazionale di lingua e letteratura (16.5.2011), http://www.patrialetteratura.com/il-manifesto-transculturale-aggiornato/ (consultazione 15.02.2018).

Gnisci, Armando: Via della transculturazione e della gentilezza. Roma: Ensemble, 2013.

Granara, William: "Ibn Hamdīs and the Poetry of Nostalgia". In: Menocal, Maria Rosa et al. (ed.): The Literature of Al-Andalus. New York: Cambridge University Press, 2000, 388-402.

Kleinhans, Martha / Schwaderer, Richard (ed.): Transkulturelle italophone Literatur. Letteratura italofona transculturale. Würzburg: Königshausen \& Neumann, 2013. 
Le Pichon, Alain / Caronia, Letizia (ed.): Sguardi venuti da lontano. Un’indagine di Transcultura. Introd. Umberto Eco. Milano: Bompiani, 1991.

Meinhof, Ulrike Hanna / Triandafyllidou, Anna (ed.): Transcultural Europe. Cultural Policy in a Changing Europe. New York: Palgrave Macmillan, 2006.

Merlini, Raffaela / Fabiani, Daniela (ed.): Narrazioni della transculturalità. Fratture, nodi, ricomposizioni. Firenze: Cesati, 2016.

Moll, Nora: L'infinito sotto casa. Letteratura e transculturalità nell'Italia contemporanea. Bologna: Pàtron Editore, 2015.

Ortiz, Fernando: Cuban Counterpoint. Tobacco and Sugar. Trad. Harriet De Onís, introd. Bronislaw Malinowski, prologo Herminio Portell Vilá e nuova introd. Fernando Coronil. Durham/London: Duke University Press, 1995.

Papa, Michele: I poeti arabo-siciliani (IX-XI Sec.). Pref. Mario Soldati. Introd. Muftah El Sherif. Catania: Niccolò Giannotta Editore, 1973.

Reichardt, Dagmar (ed.): L'Europa che comincia e finisce: la Sicilia. Approcci transculturali alla letteratura siciliana. Beiträge zur transkulturellen Annäherung an die sizilianische Literatur. Contributions to a Transcultural Approach to Sicilian Literature. Con una premessa di Dagmar Reichardt, in collaborazione con Anis Memon, Giovanni Nicoli e Ivana Paonessa. Frankfurt a.M. et al.: Lang, 2006.

Reichardt, Dagmar: "Costruzioni transculturali: costanti europee e scatti d'interscambio dinamico nei testi poetici di Freni, Bonaviri, Balistreri e Scollo". In: Van den Bossche, Bart et al. (ed.): "Innumerevoli contrasti d'innesti”: la poesia del Novecento (e altro). Miscellanea in onore di Franco Musarra. Vol. 1. Firenze/Leuven: Cesati/Leuven University Press, 2007, 339-355.

Reichardt, Dagmar: "On the Theory of a Transcultural Francophony. The Concept of Wolfgang Welsch and its Didactic Interest". In: Transnational '900. Novecento transnazionale. Letterature, arti e culture / Transnational 20th Century. Literatures, Arts and Cultures 1,1 (marzo 2017), 40-56, http://ojs.uniroma1.it/index.php/900Transnazionale/article/view/13821/13588 (consultazione 29.05.2018).

Reichardt, Dagmar: "Radicata a Roma: la svolta transculturale nella scrittura italofona nomade di Jhumpa Lahiri”. In: Geat, Marina (ed.): Il pensiero letterario come fondamento di una testa ben fatta. Roma: Roma TRE Press, 2017, 219-247, http://romatrepress.uniroma3.it/ojs/index.php/ pensiero/article/view/632 (consultazione 15.02.2018).

Reichardt, Dagmar: "Weltmusikalisches Crossover - fließende Gefühle. Etta Scollo zum 60. Geburtstag”. In: Kultur-Port.de (26.5.2018), https://www.kultur-port.de/index.php/blog/musik/15038etta-scollo-zum-60-geburtstag-weltmusik-crossover-sizilien.html (consultazione 29.05.2018).

Reichardt, Dagmar / Moll, Nora (ed.): Italia transculturale. Il sincretismo italofono come modello eterotopico. Con la collaborazione di Donatella Brioschi. Firenze: Cesati, 2018 [in stampa].

Scego, Igiaba: La mia casa è dove sono. Milano: Rizzoli, 2010.

Sciascia, Leonardo: La Sicilia come metafora. Intervista di Marcelle Padovani. Milano: Mondadori, 1979.

Sorgi, Marcello: La testa ci fa dire. Dialogo con Andrea Camilleri. Palermo: Sellerio, 2000.

Vattimo, Gianni / Welsch, Wolfgang (ed.): Medien-Welten Wirklichkeiten. München: Fink, 1998. 
Welsch, Wolfgang: “Transculturality: The Puzzling Form ofCultures Today”. In: Featherstone, Mike/ Lash, Scott (ed.): Spaces of Culture: City, Nation, World. London: Sage, 1999, 194-213.

Welsch, Wolfgang: Transkulturalität: Realität - Geschichte-Aufgabe. Wien: New Academic Press, 2017.

Wimmer, Franz Martin: "Thesen, Bedingungen und Aufgaben interkulturell orientierter Philosophie”. In: Polylog. Forum für interkulturelle Philosophie 1 (2000), https://them.polylog.org/1/fwfde.htm (consultazione 13.07.2018).

\section{Discografia}

Balistreri, Rosa: La cantatrice del Sud. RCA International INTI 1552, 1973 (LP) [riedizione del disco La voce della Sicilia (Tauro Record, 1967), registrato in seguito, in formato CD, sotto il titolo Rosa Balistreri (Teatro del Sole, 1996)].

Balistreri, Rosa: Rosa canta e cunta. Rari e inediti. Teatro del Sole/Graham \& Associati TDS 07001 CNT, 2007 (CD con booklet).

Scollo, Etta: Oh Darling. Columbia 1334347, 1988 (EP).

Scollo, Etta: Casa. Mongebel (Pinpoint) MON 0052003 (CD con booklet).

Scollo, Etta: Canta Ro'. Omaggio a Rosa Balistreri. Insieme all'Orchestra Sinfonica Siciliana. Premium Records PRE 006, 2005 (concerto dal vivo; CD con DVD e booklet).

Scollo, Etta: Canta Ro' in Trio. Insieme a Frank Wulff, Hinrich Dageför e Sebastiano Scollo. Premium Records PRE 007, 2006 (concerti dal vivo; CD con booklet).

Scollo, Etta: Les Siciliens. Premium Records PRE 019, 2007 (concerto dal vivo, CD).

Scollo, Etta: Il fiore splendente. Edel Classic 0016362BC, 2008 (CD).

Scollo, Etta: Cuoresenza. Trocadero TR 20472, 2011 (CD).

Scollo, Etta: Lunaria. Dalla favola teatrale di Vincenzo Consolo. Jazzhaus Records JHR 087, 2014 (CD con booklet).

Scollo, Etta: Scollo con cello. Tempo al tempo. Insieme a Susanne Paul. Jazzhaus Records JHR 107, 2015 (CD con booklet).

Scollo, Etta: "Sconosciuto - unbekannt" (2017), insieme a Susanne Paul, Cathrin Pfeifer e Hinrich Dageför. In: http://lampedusa361.de/installation/ (consultazione 15.2.2018). 